GLASNIK MATEMATIČKI

Vol. 41(61)(2006), 9 - 30

\title{
ON A CERTAIN FAMILY OF QUARTIC THUE EQUATIONS WITH THREE PARAMETERS
}

\author{
VOLKER ZIEGLER \\ Technische Universität Graz, Austria

\begin{abstract}
We consider the parameterized Thue equation $X^{4}-4 s X^{3} Y-(2 a b+4(a+b) s) X^{2} Y^{2}-4 a b s X Y^{3}+a^{2} b^{2} Y^{4}= \pm 1$, with $a, b \in \frac{1}{4} \mathbb{Z}$ such that $a b \in \mathbb{Z}$. By the hypergeometric method and a method of Tzanakis we find all solutions, if $s$ is large with respect to $|a|$ and $|b|$.
\end{abstract}

\section{INTRODUCTION}

Let $F \in \mathbb{Z}[X, Y]$ be a homogeneous, irreducible polynomial of degree $d \geq 3$ and $m$ a nonzero integer. Then the Diophantine equation

$$
F(X, Y)=m
$$

is called a Thue equation in honour of A. Thue [15] who proved that Diophantine equation (1.1) has only finitely many solutions $(X, Y) \in \mathbb{Z}^{2}$. The proof of this theorem is based on Thue's approximation theorem. Given $\varepsilon>0$ and an algebraic number $\alpha$ of degree $n \geq 2$, then there are only finitely many integers $p$ and $q>0$ that satisfy

$$
\left|\alpha-\frac{p}{q}\right|<q^{-n / 2-1-\varepsilon} .
$$

Since the proof of this approximation theorem is not effective we cannot solve Thue equations by exploiting the proof of Thue. However, Thue observed that his approximation theorem can be made effective, if one can find good

2000 Mathematics Subject Classification. 11D59, 11D25, 11 D09.

Key words and phrases. Diophantine equations, parameterized Thue equations, norm form equations, simultaneous Pellian equations.

The author was partially supported by the Austrian Science Foundation, project S8307-MAT. 
approximations to $\alpha$. Although Thue never stated explicitly anything like this, Thue [16] actually solved the family of Thue equations

$$
(a+1) X^{n}-a Y^{n}=1,
$$

where $n \geq 3$ is a prime and $a$ is suitable large with respect to $n$. He obtained his good approximations by considering suitable differential equations and their related hypergeometric functions. Mahler [12] was the first who stated results on effective measurement of algebraic numbers. For Thue equations of degree 3 Chudnovsky [8] gives a detailed study on the Thue-Siegel method.

In the 60's of the previous century, Baker $[1,3]$ considered linear forms of logarithms. In a further paper [2], he used his results on linear forms in order to show how Thue equations can be solved algorithmically. Using Baker's method, Bugeaud and Győry [7] computed upper bounds for the solutions of a single Thue equation. These bounds only depend on the regulator, the degree of the related number field and the degree of the Thue equation. Also efficient algorithms have been developed by several authors. The most famous are from Tzanakis and de Weger [18] and from Bilu and Hanrot [6].

In 1990 Thomas [14] considered the family

$$
X^{3}-(n-1) X^{2} Y-(n+2) X Y^{2}-Y^{3}=1,
$$

where $n$ is some parameter running through all positive integers. This was the first time that a family of Thue equations with positive discriminant has been solved.

Another practical approach to solve Thue equations is the method of Tzanakis [17] who showed how to reduce quartic Thue equations of certain type to a system of Pellian equations. Using the method of Tzanakis, Dujella and Jadrijević [9] solved the parametrized Thue equation

$$
X^{4}-4 n X^{3} Y+(6 n+2) X^{2} Y^{2}+4 n X Y^{3}+Y^{4}=1
$$

by reducing it to the system

$$
\begin{aligned}
(2 n+1) U^{2}-2 n V^{2} & =1, \\
(n-2) U^{2}-n Z^{2} & =-2
\end{aligned}
$$

of Pellian equations. They solved this system for all rational integers $n \geq 4$ by the method of Baker and Davenport (cf. [4]) combined with the congruence method (cf. [9]) and a result of Bennett [5] about simultaneous approximations of square roots. By a refinement of their method, Dujella and Jadrijević (cf. [10]) solved the Thue inequality

$$
\left|X^{4}-4 n X^{3} Y+(6 n+2) X^{2} Y^{2}+4 n X Y^{3}+Y^{4}\right| \leq 6 n+4
$$

The aim of this paper is to solve following family of Thue equations:

$$
X^{4}-4 s X^{3} Y-(2 a b+4 s(a+b)) X^{2} Y^{2}-4 a b s X Y^{3}+a^{2} b^{2} Y^{4}=\mu,
$$


where $\mu \in\{1,-1\}, a, b \in \frac{1}{4} \mathbb{Z}$, with $a \neq b$ and $0 \neq a b \in \mathbb{Z}$ and $s \in \mathbb{Z}$ large with respect to $|a|$ and $|b|$. Observe that for $a=-2, b=1 / 2$ and $\mu=1$ we obtain equation (1.2). In particular we prove the following theorem:

TheOrem 1.1. Let $(X, Y)$ be a solution to Thue equation (1.4) with $s \in \mathbb{Z}$, $a, b \in \frac{1}{4} \mathbb{Z}, a \neq b,|a| \geq|b|$ and $0 \neq a b \in \mathbb{Z}$ and suppose $s>7.23 \cdot 10^{10}|a|^{\frac{29+\sqrt{241}}{4}}$. Then necessarily $\mu=1$. Furthermore, the only solutions are $(X, Y)=( \pm 1,0)$, $(X, Y)=(0, \pm 1)$ if $a b= \pm 1$ or those listed in Table 1.

TABle 1. Solutions to (1.4), provided $s$ is "large".

\begin{tabular}{|r|r|r|r||r|r|r|r|}
\hline$a$ & $b$ & $X$ & $Y$ & $a$ & $b$ & $X$ & $Y$ \\
\hline$-\frac{17}{4}$ & -4 & \pm 4 & \pm 1 & -4 & $-\frac{15}{4}$ & \pm 4 & \pm 1 \\
$\frac{17}{4}$ & 4 & \pm 4 & $\mp 1$ & 4 & $\frac{15}{4}$ & \pm 4 & $\mp 1$ \\
$-\frac{5}{2}$ & -2 & \pm 2 & \pm 1 & -2 & $-\frac{3}{2}$ & \pm 2 & \pm 1 \\
$\frac{5}{2}$ & 2 & \pm 2 & $\mp 1$ & 2 & $\frac{3}{2}$ & \pm 2 & $\mp 1$ \\
-2 & -1 & \pm 1 & \pm 1 & & & & \\
2 & 1 & \pm 1 & $\mp 1$ & & & & \\
\hline
\end{tabular}

Observe that there is no solution in the case of $\mu=-1$ and $s$ sufficiently large. Furthermore it is no restriction to assume that $|a| \geq|b|$, since equation (1.4) is symmetric in $a$ and $b$.

The rest of the paper is organized as follows. In Section 2 we present some preliminary results and investigate asymptotic expansions of the relevant roots. How to reduce Thue equation (1.4) to a system of Pellian equations is demonstrated in Section 3. For solutions to this system we will find an upper bound by the hypergeometric method (cf. Section 4). In order to obtain a lower bound we will use Padé approximations in Section 5. The proof of Theorem 1.1 will be finished in Section 6 , where we consider the remaining case $|Y|=1$. In the last section we will state special cases of Theorem 1.1, where $a, b \in \frac{1}{2} \mathbb{Z}$, respectively $a, b \in \mathbb{Z}$, and give some examples.

\section{Preliminaries}

We start with the norm form equation

$$
\mathrm{N}_{\mathbb{Q}}^{K}(X+\alpha Y)= \pm 1,
$$


where

$$
\alpha=\sqrt{s(s+a)}+\sqrt{s(s+b)}+\sqrt{(s+a)(s+b)}+s,
$$

with $s \in \mathbb{Z}$ and $a, b \in \frac{1}{4} \mathbb{Z}$, such that $a b \in \mathbb{Z},|a| \geq|b|$ and $a \neq b$. Obviously, $\alpha$ is an element of the compositum $K:=\mathbb{Q}(\sqrt{s(s+a)}) \mathbb{Q}(\sqrt{s(s+b)})$. In any case, $K$ is Galois, since $K$ is the compositum of two fields that are Galois over $\mathbb{Q}$. If $s>\max (|a|,|b|)$, then $K$ is a real field and moreover $K$ is quartic if and only if none of the quantities $s(s+a), s(s+b)$ and $(s+a)(s+b)$ is a perfect square.

Lemma 2.1. Assume $s>(2|a|+1 / 4)^{2}$. Then $K$ is Galois, real and quartic with Galois group $G \simeq \mathbb{Z} / 2 \mathbb{Z} \times \mathbb{Z} / 2 \mathbb{Z}$.

Proof. From the discussion above we know that $K$ is Galois and real. Let us assume $(s+a)(s+b)$ is not a perfect square. Then $\mathbb{Q}(\sqrt{s(s+a)}) \cap$ $\mathbb{Q}(\sqrt{s(s+b)})=\mathbb{Q}$ and therefore $K$ is quartic and we know from Galois theory that the Galois group is of the wanted form (cf. [11, Chapter VI, Theorem 1.14]). So we are left to prove that neither $s(s+a), s(s+b)$ nor $(s+a)(s+b)$ is a perfect square.

Assume $(s+a)(s+b)$ is a perfect square. From the assumptions on $a$ and $b$ we find that $4(s+a)(s+b)$ is the square of an integer. On the other hand, we have

$$
\begin{aligned}
(2 s+(a+b))^{2} & =4(s+a)(s+b)+(a-b)^{2} \\
& >4(s+a)(s+b), \\
(2 s+(a+b)-1 / 4)^{2} & =4(s+a)(s+b)-\left(s+\frac{a+b}{2}-(a-b)^{2}-\frac{1}{16}\right) \\
& \leq 4(s+a)(s+b)-\left(s-(2|a|+1 / 4)^{2}\right) \\
& <4(s+a)(s+b),
\end{aligned}
$$

a contradiction, hence $(s+a)(s+b)$ is not a perfect square. Similarly we find

$$
\begin{aligned}
(2 s+a)^{2} & >4 s(s+a), \\
(2 s+a-1 / 4)^{2} & <4 s(s+a),
\end{aligned}
$$

and

$$
\begin{aligned}
(2 s+b)^{2} & >4 s(s+b), \\
(2 s+b-1 / 4)^{2} & <4 s(s+b) .
\end{aligned}
$$

Therefore neither of $s(s+a), s(s+b)$ nor $(s+a)(s+b)$ is a perfect square.

\section{口}

Because of Lemma 2.1 we assume for the rest of the paper that $s>(2|a|+$ $1 / 4)^{2}$. Moreover we immediately obtain from Galois theory the conjugates 
$\alpha_{1}, \ldots, \alpha_{4}$ of $\alpha$ :

$$
\begin{aligned}
\alpha=\alpha_{1} & =s+\sqrt{s(s+a)}+\sqrt{s(s+b)}+\sqrt{(s+a)(s+b)} \\
\alpha_{2} & =s-\sqrt{s(s+a)}+\sqrt{s(s+b)}-\sqrt{(s+a)(s+b)} \\
\alpha_{3} & =s+\sqrt{s(s+a)}-\sqrt{s(s+b)}-\sqrt{(s+a)(s+b)} \\
\alpha_{4} & =s-\sqrt{s(s+a)}-\sqrt{s(s+b)}+\sqrt{(s+a)(s+b)}
\end{aligned}
$$

Therefore we are able to compute the minimal polynomial $f \in \mathbb{Q}[X]$ of $\alpha$ :

$$
f(X):=X^{4}-4 s X^{3}-(2 a b+4(a+b) s) X^{2}-4 a b s X+a^{2} b^{2},
$$

i.e. norm form equation (2.1) is equivalent to Thue equation

$$
F(X, Y):=X^{4}-4 s X^{3} Y-(2 a b+4 s(a+b)) X^{2} Y^{2}-4 a b s X Y^{3}+a^{2} b^{2} Y^{4}= \pm 1 .
$$

Furthermore we have proved that $\alpha$ is an algebraic integer.

Next we want to investigate the asymptotic of the $\alpha$ 's as $s \rightarrow \infty$. Because of the structure of the $\alpha$ 's, we only have to consider the asymptotic of $\sqrt{s(s+a)}, \sqrt{s(s+b)}$ and $\sqrt{(s+b)(s+a)}$. The following expansion is well known

$$
\sqrt{1+\frac{a}{s}}=\sum_{n=0}^{\infty}\left(\begin{array}{c}
1 / 2 \\
n
\end{array}\right) \frac{a^{n}}{s^{n}}
$$

and it is valid for $s>|a|$. This implies

$$
\begin{aligned}
\sqrt{s(s+a)} & =\sum_{n=0}^{\infty}\left(\begin{array}{c}
1 / 2 \\
n
\end{array}\right) \frac{a^{n}}{s^{n-1}} \\
\sqrt{s(s+b)} & =\sum_{n=0}^{\infty}\left(\begin{array}{c}
1 / 2 \\
n
\end{array}\right) \frac{b^{n}}{s^{n-1}} \\
\sqrt{(s+b)(s+a)} & =\sum_{n=0}^{\infty} \sum_{k=0}^{n}\left(\begin{array}{c}
1 / 2 \\
k
\end{array}\right)\left(\begin{array}{c}
1 / 2 \\
n-k
\end{array}\right) \frac{a^{k} b^{n-k}}{s^{n-1}}
\end{aligned}
$$

where all three expansions are valid if $s>|a|$. The following variant of the usual $O$-notation is used. For two functions $g(s)$ and $h(s)$ we write $g(s)=$ $L(h(s))$ if $|g(s)| \leq h(s)$. This notation is used in the middle of an expression in the same way as it is usually done with $O$-notation. With this $L$-notation we obtain

$$
\begin{aligned}
\sqrt{s(s+a)} & =\sum_{n=0}^{N}\left(\begin{array}{c}
1 / 2 \\
n
\end{array}\right) \frac{a^{n}}{s^{n-1}}+L\left(\sum_{n=N+1}^{\infty}\left|\left(\begin{array}{c}
1 / 2 \\
n
\end{array}\right)\right| \frac{|a|^{n}}{s^{n-1}}\right) \\
& =\sum_{n=0}^{N}\left(\begin{array}{c}
1 / 2 \\
n
\end{array}\right) \frac{a^{n}}{s^{n-1}}+L\left(\left|\left(\begin{array}{c}
1 / 2 \\
N+1
\end{array}\right)\right| \frac{|a|^{N+1}}{s^{N}} \frac{s}{s-|a|}\right) .
\end{aligned}
$$


For an exact asymptotic of $\sqrt{(s+b)(s+a)}$ we consider the $N$-th coefficient of its expansion. By elementary calculations we observe that

$$
C(N):=\sum_{k=0}^{N}\left|\left(\begin{array}{c}
1 / 2 \\
k
\end{array}\right)\left(\begin{array}{c}
1 / 2 \\
N-k
\end{array}\right)\right|=\left\{\begin{array}{cl}
4\left|\left(\begin{array}{c}
1 / 2 \\
N
\end{array}\right)\right| & \text { if } N \geq 2 \\
1 & \text { if } N=0,1
\end{array}\right.
$$

Since $|C(N)|$ is decreasing with $N$, this implies

$$
\begin{aligned}
\sqrt{(s+b)(s+a)}= & \sum_{n=0}^{N} \sum_{k=0}^{n}\left(\begin{array}{c}
1 / 2 \\
k
\end{array}\right)\left(\begin{array}{c}
1 / 2 \\
n-k
\end{array}\right) \frac{a^{k} b^{n-k}}{s^{n-1}} \\
& +L\left(\sum_{n=N+1}^{\infty} C(n) \frac{|a|^{n}}{s^{n-1}}\right) \\
= & \sum_{n=0}^{N} \sum_{k=0}^{n}\left(\begin{array}{c}
1 / 2 \\
k
\end{array}\right)\left(\begin{array}{c}
1 / 2 \\
n-k
\end{array}\right) \frac{a^{k} b^{n-k}}{s^{n-1}} \\
& +L\left(C(N+1) \frac{|a|^{N+1}}{s^{N}} \frac{s}{s-|a|}\right) .
\end{aligned}
$$

\section{From Thue equations to Pellian equations}

In 1993, Tzanakis [17] considered Thue equations of the form

$$
F(X, Y):=a_{0} X^{4}+4 a_{1} X^{3} Y+6 a_{2} X^{2} Y^{2}+4 a_{3} X Y^{3}+a_{4} Y^{4}=m
$$

such that $F(X, Y) \in \mathbb{Z}[X, Y], m \in \mathbb{Z}$ and $a_{0}>0$. Furthermore the corresponding number field $K$ has to be Galois and non-cyclic. If we assume $K$ is not totally complex, i.e. there is some real root of $F(X, 1)$, then $K$ is the compositum of two real quadratic fields. Furthermore the equation

$$
4 \sigma^{3}-g_{2} \sigma-g_{3}=0
$$

has three distinct rational roots $\sigma_{1}, \sigma_{2}$ and $\sigma_{3}$; here $g_{2}$ and $g_{3}$ are invariants of the following form:

$$
g_{2}=a_{0} a_{4}-4 a_{1} a_{3}+3 a_{2}^{2}, \quad g_{3}=\operatorname{det}\left(\begin{array}{ccc}
a_{0} & a_{1} & a_{2} \\
a_{1} & a_{2} & a_{3} \\
a_{2} & a_{3} & a_{4}
\end{array}\right) .
$$

Let $H(X, Y)$ and $G(X, Y)$ be the quartic and sextic covariants of $F(X, Y)$ respectively (cf. [13]), i.e.

$$
H(X, Y)=-\frac{1}{144}\left|\begin{array}{cc}
\frac{\partial^{2} f}{\partial X^{2}} & \frac{\partial^{2} f}{\partial X \partial Y} \\
\frac{\partial^{2} f}{\partial Y \partial X} & \frac{\partial^{2} f}{\partial Y^{2}}
\end{array}\right|, \quad G(X, Y)=-\frac{1}{8}\left|\begin{array}{cc}
\frac{\partial f}{\partial X} & \frac{\partial f}{\partial Y} \\
\frac{\partial H}{\partial X} & \frac{\partial H}{\partial Y}
\end{array}\right| .
$$


We have

$$
H(X, Y) \in \frac{1}{48} \mathbb{Z}[X, Y], \quad G(X, Y) \in \frac{1}{96} \mathbb{Z}[X, Y]
$$

and (cf. [13, Chapter 25, Theorem 1])

$$
4 H^{3}-g_{2} H f^{2}-g_{3} f^{3}=G^{2} .
$$

Let us put now

$$
H=\frac{1}{48} H_{0}, \quad G=\frac{1}{96} G_{0}, \quad \sigma_{i}=\frac{1}{12} r_{i}(i=1,2,3) .
$$

Then $H_{0}, G_{0} \in \mathbb{Z}[X, Y]$ and $r_{i} \in \mathbb{Z}$. In view of equation (3.3) we have

$$
\left(H_{0}-4 r_{1} f\right)\left(H_{0}-4 r_{2} f\right)\left(H_{0}-4 r_{3} f\right)=3 G_{0}^{2} .
$$

Since $H$ and $f$ are relatively prime (cf. [17, Proposition 1]) there exist squarefree integers $k_{1}, k_{2}$ and $k_{3}$ and quadratic forms $G_{i} \in \mathbb{Z}[X, Y], i=1,2,3$ such that

$$
H_{0}-4 r_{i} f=k_{i} G_{i}^{2} \quad i=1,2,3 .
$$

If $(X, Y)$ is a solution to (3.1), we obtain from identity (3.4) the system

$$
k_{2} G_{2}^{2}-k_{1} G_{1}^{2}=4\left(r_{1}-r_{2}\right) m, \quad k_{3} G_{3}^{2}-k_{1} G_{1}^{2}=4\left(r_{1}-r_{3}\right) m
$$

of Pellian equations.

Applying this procedure to Thue equation (2.3) we obtain

$$
\begin{gathered}
a_{0}=1, \quad a_{1}=-s, \quad a_{2}=-\frac{a b+2 s(a+b)}{3}, \quad a_{3}=-s a b, \quad a_{4}=a^{2} b^{2} ; \\
g_{2}=a^{2} b^{2}-4 a b s^{2}+\frac{1}{3}(a b+2 s(a+b))^{2}, \\
g_{3}=-\frac{4}{27}(2 a b+s(a+b))(a b+s(2 b-a))(a b+s(2 a-b)) ; \\
\sigma_{1}=-\frac{1}{3}(2 a b+a s+b s), \quad \sigma_{2}=\frac{1}{3}(-b s+a b+2 a s), \\
\sigma_{3}=\frac{1}{3}(-a s+a b+2 b s) ; \\
G_{1}=X^{2}-a b Y^{2}, \quad G_{2}=X^{2}+2 a X Y+a b Y^{2}, \quad G_{3}=X^{2}+2 b X Y+a b Y^{2} ; \\
k_{1}=48(a+s)(b+s), \quad k_{2}=48 s(b+s), \quad k_{3}=48 s(a+s) .
\end{gathered}
$$

This yields the system

$$
(a+s) U^{2}-s V^{2}=\mu a, \quad(b+s) U^{2}-s Z^{2}=\mu b,
$$

with

$U=X^{2}-a b Y^{2}, \quad V=X^{2}+2 a X Y+a b Y^{2}, \quad Z=X^{2}+2 b X Y+a b Y^{2}$ and $\mu= \pm 1$. 


\section{Hypergeometric method}

In this section we want to find an upper bound for $|U|$ if $(U, V, Z)$ is a solution to system (3.6). Let us first observe that if $(U, V, Z)$ is a solution to (3.6), then also $( \pm U, \pm V, \pm Z$ ) is a solution to (3.6). Therefore we may assume without loss of generality $U, V, Z \geq 0$. Furthermore $U=0$ yields $s V^{2}= \pm a$ and, since we assume $s>(2|a|+1 / 4)^{2}$, we have $|V|<1$, hence $V=0$ and $a=0$, a contradiction. Similar arguments apply to $V$ and $Z$, therefore we may assume $U, V, Z>0$. In order to prove an upper bound for $|U|$ we will discuss first some approximation properties of solutions $(U, V, Z)$ to $(3.6)$.

Lemma 4.1. Let $(U, V, Z)$ be a solution to system (3.6) with $U, V, Z>0$. Then

$$
\begin{aligned}
& \left|\frac{V}{U}-\sqrt{1+\frac{a}{s}}\right| \leq \frac{|a|}{U^{2}} \sqrt{\frac{1}{(s+a) s}} ; \\
& \left|\frac{Z}{U}-\sqrt{1+\frac{b}{s}}\right| \leq \frac{|b|}{U^{2}} \sqrt{\frac{1}{(s+b) s}} .
\end{aligned}
$$

Proof. We only prove the first inequality. The proof of the second inequality is analogous. One just has to replace $Z$ by $V$ and $b$ by $a$. Since $U, V>0$ we have

and therefore

$$
V+U \sqrt{1+\frac{a}{s}} \geq U \sqrt{1+\frac{a}{s}}
$$

$$
\begin{aligned}
\left|V-U \sqrt{1+\frac{a}{s}}\right| & =\frac{\left|V-U \sqrt{1+\frac{a}{s}}\right|\left|V+U \sqrt{1+\frac{a}{s}}\right|}{\left|V+U \sqrt{1+\frac{a}{s}}\right|} \\
& \leq \frac{|a|}{s U \sqrt{1+a / s}}=\frac{|a|}{U} \sqrt{\frac{1}{(s+a) s}} .
\end{aligned}
$$

Division with $U$ yields the lemma.

Since we assume $|a| \geq|b|$ the lemma above shows that

$$
\max \left(\left|\frac{V}{U}-\sqrt{1+\frac{a}{s}}\right|,\left|\frac{Z}{U}-\sqrt{1+\frac{b}{s}}\right|\right) \leq \frac{|a|}{U^{2}} \sqrt{\frac{1}{(s+a) s}} .
$$

Hence we have found a good simultaneous approximation to $\sqrt{1+\frac{a}{s}}$ and $\sqrt{1+\frac{b}{s}}$. The following discussion will show that this approximation is in some sense too good. We start with a theorem of Bennett [5, Theorem 3.2].

THEOREM 4.2. If $a_{i}, p_{i}, q$ and $N$ are integers for $0 \leq i \leq 2$ with $a_{0}<a_{1}<$ $a_{2}, a_{j}=0$ for some $0 \leq j \leq 2$, q nonzero and $N>M^{9}$, where $M=\max _{i=0,1,2}\left|a_{i}\right|$, 
then we have

$$
\max _{i=0,1,2}\left(\left|\sqrt{1+\frac{a_{i}}{N}}-\frac{p_{i}}{q}\right|\right)>(130 N \Upsilon)^{-1} q^{-\lambda}=c^{-1} q^{-\lambda},
$$

where

and

$$
\lambda=1+\frac{\log (33 N \Upsilon)}{\log \left(1.7 N^{2} \prod_{0 \leq i<j \leq 2}\left(a_{i}-a_{j}\right)^{-2}\right)}
$$

$$
\Upsilon= \begin{cases}\frac{\left(a_{2}-a_{0}\right)^{2}\left(a_{2}-a_{1}\right)^{2}}{2 a_{2}-a_{0}-a_{1}} & \text { if } a_{2}-a_{1} \geq a_{1}-a_{0}, \\ \frac{\left(a_{2}-a_{0}\right)^{2}\left(a_{1}-a_{0}\right)^{2}}{a_{1}+a_{2}-2 a_{0}} & \text { if } a_{2}-a_{1}<a_{1}-a_{0} .\end{cases}
$$

We want to apply Theorem 4.2 to $a_{i}=a^{\prime}$ and $a_{j}=b^{\prime}$ with some $i, j \in$ $\{0,1,2\}, i \neq j, a=a^{\prime} / 4, b=b^{\prime} / 4$ and $N=4 s$. First let us estimate $\Upsilon$. Therefore we have to distinguish between 6 cases (remind that we always assume $|a| \geq|b|$, hence $\left.\left|a^{\prime}\right| \geq\left|b^{\prime}\right|\right)$.

- Suppose $a^{\prime}>b^{\prime}>0$ and $a^{\prime}-b^{\prime} \geq b^{\prime}-0$. Then we have $a^{\prime} \geq 2 b^{\prime}$ and

$$
\Upsilon=\frac{\left(a^{\prime}-0\right)^{2}\left(a^{\prime}-b^{\prime}\right)^{2}}{2 a^{\prime}-b^{\prime}-0}=\frac{a^{\prime 2}\left(a^{\prime}-b^{\prime}\right)^{2}}{2 a^{\prime}-b^{\prime}} \leq \frac{2}{3}\left|a^{\prime}\right|^{3} .
$$

- Let $a^{\prime}>b^{\prime}>0$ and $a^{\prime}-b^{\prime}<b^{\prime}-0$. The last inequality is $a^{\prime}<2 b^{\prime}$ and therefore

$$
\Upsilon=\frac{\left(a^{\prime}-0\right)^{2}\left(b^{\prime}-0\right)^{2}}{b^{\prime}+a^{\prime}-20}=\frac{a^{\prime 2} b^{\prime 2}}{a^{\prime}+b^{\prime}} \leq \frac{2}{3}\left|a^{\prime}\right|^{3} .
$$

- Assume $a^{\prime}>0>b^{\prime}$. Since $\left|a^{\prime}\right| \geq\left|b^{\prime}\right|$ we have $a_{2}-a_{1} \geq a_{1}-a_{0}$, i.e.

$$
\Upsilon=\frac{\left(a^{\prime}-b^{\prime}\right)^{2}\left(a^{\prime}-0\right)^{2}}{2 a^{\prime}-b^{\prime}-0}=\frac{\left(a^{\prime}-b^{\prime}\right)^{2} a^{\prime 2}}{2 a^{\prime}-b^{\prime}} \leq \frac{4}{3}\left|a^{\prime}\right|^{3} .
$$

- Provided $b^{\prime}>0>a^{\prime}$ we have $a_{2}-a_{1} \leq a_{1}-a_{0}$, hence in both cases "<" and "=" we find

$$
\Upsilon=\frac{\left(b^{\prime}-a^{\prime}\right)^{2}\left(0-a^{\prime}\right)^{2}}{0+b^{\prime}-2 a^{\prime}}=\frac{\left(b^{\prime}-a^{\prime}\right)^{2} a^{\prime 2}}{-2 a^{\prime}+b^{\prime}} \leq \frac{4}{3}\left|a^{\prime}\right|^{3} .
$$

- In the case of $0>b^{\prime}>a^{\prime}$ and $0-b^{\prime} \geq b^{\prime}-a^{\prime}$ we have $2\left|b^{\prime}\right| \geq\left|a^{\prime}\right|$ and obtain following estimation

$$
\Upsilon=\frac{\left(0-b^{\prime}\right)^{2}\left(0-a^{\prime}\right)^{2}}{2 \cdot 0-b^{\prime}-a^{\prime}}=\frac{b^{\prime 2} a^{\prime 2}}{-b^{\prime}-a^{\prime}} \leq \frac{2}{3}\left|a^{\prime}\right|^{3} .
$$

- At last we consider the case $0>b^{\prime}>a^{\prime}$ and $0-b^{\prime}<b^{\prime}-a^{\prime}$. Therefore $2\left|b^{\prime}\right|<\left|a^{\prime}\right|$ and

$$
\Upsilon=\frac{\left(0-a^{\prime}\right)^{2}\left(b^{\prime}-a^{\prime}\right)^{2}}{b^{\prime}+0-2 a^{\prime}}=\frac{a^{\prime 2}\left(b^{\prime}-a^{\prime}\right)^{2}}{-2 a^{\prime}+b^{\prime}} \leq \frac{2}{3}\left|a^{\prime}\right|^{3} .
$$


All cases together yield the estimation $\Upsilon \leq \frac{4}{3}\left|a^{\prime}\right|^{3}=\frac{256}{3}|a|^{3}$. Hence we have

$$
c \leq c^{\prime}:=\frac{133120}{3} s|a|^{3}, \quad \lambda \leq \lambda^{\prime}=1+\frac{\log \left(11264 s|a|^{3}\right)}{\log \left(27.2 s^{2}\right)-\log \left(16384|a|^{6}\right)} .
$$

We want to have $\lambda<2$. Therefore we consider the inequality

$$
1<\frac{\log \left(11264 s|a|^{3}\right)}{\log \left(27.2 s^{2}\right)-\log \left(16384|a|^{6}\right)}
$$

or equivalently

$$
\log 11264+\log s+3 \log |a|<\log 27.2+2 \log s-\log 16384-6 \log |a| .
$$

The last inequality holds if $\log s>\log \frac{184549376}{27.2}+9 \log |a|$, i.e. $s>\frac{184549376}{27.2}|a|^{9}$. Therefore we will assume for the rest of this section $s>\frac{184549376}{27.2}|a|^{9}$. The assumption $N>M^{9}$, i.e. $4 s>4^{9}|a|^{9}$ is now fulfilled and by an application of Theorem 4.2, together with Lemma 4.1, we obtain

$$
c^{\prime-1} U^{-\lambda^{\prime}}<\max \left(\left|\frac{V}{U}-\sqrt{1+\frac{a}{s}}\right|,\left|\frac{Z}{U}-\sqrt{1+\frac{b}{s}}\right|\right) \leq \frac{|a|}{U^{2}} \sqrt{\frac{1}{(s+a) s}} .
$$

Taking logarithms and solving for $\log U$ yields

$$
\begin{aligned}
\log U & <\frac{1}{2-\lambda^{\prime}}\left(\log c^{\prime}+\log |a|-\frac{1}{2} \log ((s+a) s)\right) \\
& <\frac{1}{2-\lambda^{\prime}}\left(\log \frac{133120}{3}+\log s+4 \log |a|-\frac{1}{2}\left(2 \log s-\frac{27.2}{184549376}\right)\right) \\
& <\frac{1}{2-\lambda^{\prime}}(4 \log |a|+10.71) .
\end{aligned}
$$

Let us assume $s>c_{0}|a|^{9+r}$. Then we have

$$
\begin{aligned}
\frac{1}{2-\lambda^{\prime}} & =\frac{1}{1-\frac{\log \left(11264 s|a|^{3}\right)}{\log \left(27.2 s^{2}\right)-\log \left(16384|a|^{6}\right)}} \\
& =\frac{\log \left(27.2 s^{2}\right)-\log \left(16384|a|^{6}\right)}{\log \left(27.2 s^{2}\right)-\log \left(16384|a|^{6}\right)-\log \left(11264 s|a|^{3}\right)} \\
(4.3) & =\frac{2 \log s-6 \log |a|+\log \left(\frac{27.2}{16384}\right)}{\log s-9 \log |a|+\log \left(\frac{27.2}{16384 \cdot 11264}\right)} \\
& <\frac{\left(2-\frac{6}{9+r}\right) \log s+\frac{6}{9+r} \log c_{0}+\log \left(\frac{27.2}{16384}\right)}{\left(1-\frac{9}{9+r}\right) \log s+\frac{9}{9+r} \log c_{0}+\log \left(\frac{27.2}{16384 \cdot 11264}\right)} \\
& =\frac{12+2 r}{r}-\frac{9+r}{r} \cdot \frac{12 \log c_{0}-r \log \left(\frac{1299227607040}{17}\right)-12 \log \left(\frac{115343360}{17}\right)}{r \log s+9 \log c_{0}-(9+r) \log \left(\frac{115343360}{17}\right)} .
\end{aligned}
$$


The inequality above holds, since $\frac{1}{2-\lambda^{\prime}}$ is as function increasing with $|a|$. Let us consider the second fraction of the last line in (4.3). As one can easily compute, the numerator is $\geq 0$ if

$$
c_{0} \geq 2^{21+\frac{31 r}{12}}\left(\frac{17}{5}\right)^{-1-\frac{r}{12}} 11^{1+\frac{r}{6}} .
$$

If $c_{0}$ fulfills this inequality another computation shows that the denominator of this fraction is positive for $s \geq 1$. Hence we have proved

$$
\frac{1}{2-\lambda^{\prime}}<\frac{12+2 r}{r}
$$

provided (4.4) holds. By combining (4.2) and (4.5) we obtain

$$
\begin{aligned}
\log U & <\frac{48+8 r}{r} \log |a|+10.71 \frac{12+2 r}{r} \\
& <\frac{48+8 r}{(9+r) r} \log s-\frac{48+8 r}{(9+r) r} \log c_{0}+10.71 \frac{6+r}{r} .
\end{aligned}
$$

Next we want to find an upper bound for $|Y|$. First let us assume $a b<0$. Then we have

$$
U=X^{2}-a b Y^{2} \geq Y^{2}
$$

and therefore we find $\log |Y| \leq \frac{1}{2} \log |U|$. Let now $a b>0$. Then

$$
Z=X^{2}+2 b X Y+a b Y^{2}=(X+b Y)^{2}+\left(a b-b^{2}\right) Y^{2} \geq \frac{3}{4} Y^{2} .
$$

On the other hand, the second Pellian equation of (3.6) yields

$Z^{2}=\left(1+\frac{b}{s}\right) U^{2} \pm \frac{b}{s}<U^{2}\left(1+\frac{27.2}{184549376}\right)+\frac{27.2}{184549376}<(U \cdot 1.0001)^{2}$,

hence

$$
\log |Y|<0.144+\frac{1}{2} \log |U| .
$$

Therefore we have proved

Proposition 4.3. Let $(X, Y)$ be a solution to (1.4) and assume $s>$ $c_{0}(r)|a|^{9+r}$ with $r>0$ and

$$
c_{0}(r):=2^{21+\frac{31 r}{12}}\left(\frac{17}{5}\right)^{-1-\frac{r}{12}} 11^{1+\frac{r}{6}}
$$

Then

$$
\log |Y|<\frac{24+4 r}{(9+r) r} \log s-\frac{24+4 r}{(9+r) r} \log c_{0}(r)+10.71 \frac{12+2 r}{r}+0.144 .
$$




\section{Approximation properties of $\alpha$}

In the previous section we have found an upper bound for $\log |Y|$ if $s$ is large with respect to $|a|$ and $|b|$. In this section we find a lower bound for $|Y|$ provided $|Y|>1$. This bound will be found by using approximation properties of the roots $\alpha_{i}, 1 \leq i \leq 4$. We further assume $s>\frac{184549376}{27.2}|a|^{9}$. First we prove the following lemma:

Lemma 5.1. Let $(X, Y)$ be a solution to (1.4). Then at least one of the following cases occurs

$$
\begin{array}{ll}
\left|X-\alpha_{1} Y\right|<\frac{8}{63.99|Y|^{3} s^{3}} ; & \left|X-\alpha_{2} Y\right|<\frac{8}{3.99|Y|^{3} s} \\
\left|X-\alpha_{3} Y\right|<\frac{8}{2.99|Y|^{3} s} ; & \left|X-\alpha_{4} Y\right|<\frac{8}{3.99|Y|^{3} s} .
\end{array}
$$

Proof. From (2.2), (2.4) and (2.5) we find

$$
\begin{array}{ll}
\alpha_{1}=4 s+a+b+L\left(\frac{3|a|^{2}}{4 s} \cdot \frac{s}{s-|a|}\right) ; & \alpha_{2}=-a+L\left(\frac{3|a|^{2}}{4 s} \cdot \frac{s}{s-|a|}\right) ; \\
\alpha_{3}=-b+L\left(\frac{3|a|^{2}}{4 s} \cdot \frac{s}{s-|a|}\right) ; & \alpha_{4}=L\left(\frac{3|a|^{2}}{4 s} \cdot \frac{s}{s-|a|}\right) .
\end{array}
$$

Let $j$ be chosen such that $\left|X-\alpha_{j} Y\right|=\min _{i=1,2,3,4}\left|X-\alpha_{i} Y\right|$. Then we have

$$
|Y|\left|\alpha_{i}-\alpha_{j}\right| \leq\left|X-\alpha_{i} Y\right|+\left|X-\alpha_{j} Y\right| \leq 2\left|X-\alpha_{i} Y\right|
$$

Hence

$$
\left|X-\alpha_{j} Y\right|=\frac{1}{\prod_{i \neq j}\left|X-\alpha_{i} Y\right|} \leq \frac{8}{|Y|^{3} \prod_{i \neq j}\left|\alpha_{j}-\alpha_{i}\right|} .
$$

Some elementary computations yield lower bounds for $\prod_{i \neq j}\left|\alpha_{j}-\alpha_{i}\right|$ and from these we obtain the lemma.

Proposition 5.2. Let $(X, Y)$ be a solution to (1.4) with $|Y|>1$ and $s>3.5 \cdot 10^{9}|a|^{9}$. According to the four cases in Lemma 5.1 we have

$$
\begin{array}{ll}
|Y|>\frac{s^{2}}{1236.48|a|^{6}} ; & |Y|>\frac{s^{2}}{8089.6|a|^{7}} \\
|Y|>\frac{s^{2}}{8089.6|a|^{7}} ; & |Y|>\frac{s^{3}}{7107|a|^{8}} .
\end{array}
$$

Hence, in all cases we have $|Y|>\frac{s^{2}}{8089.6|a|^{7}}$. 
Proof. From (5.1) together with Lemma 5.1 we obtain

$$
\begin{array}{r}
|X-(4 s+a+b) Y|<|Y| \frac{0.751|a|^{2}}{s}+\frac{8}{63.99|Y|^{3} s^{3}}<|Y| \frac{0.752|a|^{2}}{s} \\
|X+a Y|<|Y| \frac{0.751|a|^{2}}{s}+\frac{8}{3.99|Y|^{3} s}<|Y| \frac{0.877|a|^{2}}{s} \\
|X+b Y|<|Y| \frac{0.751|a|^{2}}{s}+\frac{8}{2.99|Y|^{3} s}<|Y| \frac{0.919|a|^{2}}{s} \\
|X|<|Y| \frac{0.751|a|^{2}}{s}+\frac{8}{3.99|Y|^{3} s}<|Y| \frac{0.877|a|^{2}}{s}
\end{array}
$$

according to the four possible cases. Since the left hand sides are $\in \frac{1}{4} \mathbb{Z}$, we conclude that they vanish if

$$
|Y| \leq \frac{s}{3.008|a|^{2}}, \quad|Y| \leq \frac{s}{3.508|a|^{2}}, \quad|Y| \leq \frac{s}{3.676|a|^{2}}, \quad|Y| \leq \frac{s}{3.508|a|^{2}}
$$

Assuming (5.3) we obtain $X=(4 s+a+b) Y, X=-a Y, X=-b Y$ and $X=0$ respectively. Inserting these relations in $F(X, Y)= \pm 1$ we deduce

$$
\begin{aligned}
&|F(Y(4 s+a+b), Y)|=|Y|^{4} \mid 16 s^{2}\left(a^{2}-a b+b^{2}\right)+4 s\left(2 a^{3}+a^{2} b+a b^{2}+2 b^{3}\right) \\
&+\left(a^{4}+2 a^{3} b+3 a^{2} b^{2}+2 a b^{3}+b^{4}\right) \mid \\
&>|Y|^{4} 11.99 s^{2}|a|^{2}>1 \\
&|F(-Y a, Y)|=|Y|^{4} a^{2}(a-b)^{2} \geq|Y|^{4}>1 \\
&|F(-Y b, Y)|=|Y|^{4} b^{2}(a-b)^{2} \geq|Y|^{4} \frac{9}{16} \geq 9>1, \\
&|F(0, Y)|=|Y|^{4}>1 .
\end{aligned}
$$

In any case we get a contradiction and therefore we may assume

$$
|Y|>\frac{s}{3.008|a|^{2}}, \quad|Y|>\frac{s}{3.508|a|^{2}}, \quad|Y|>\frac{s}{3.676|a|^{2}}, \quad|Y|>\frac{s}{3.508|a|^{2}} .
$$

Now we split the proof into the four cases according to Lemma 5.1.

CASE 1: We use the approximation

$$
\alpha_{1}=4 s+a+b-\frac{a^{2}-a b+b^{2}}{4 s}+\frac{2 a^{3}-a^{2} b-a b^{2}+2 b^{3}}{16 s^{2}}+L\left(\frac{0.2347|a|^{4}}{s^{3}}\right) .
$$

Let us denote by $\bar{\alpha}_{1}$ the approximation (5.5) with omitted $L$-term. Using Padé approximations we find polynomials $P$ and $Q$ such that

$$
\begin{aligned}
\bar{\alpha}_{1} P-Q & =\frac{\left(2 a^{3}-a^{2} b-a b^{2}+2 b^{3}\right)^{2}}{16 s^{2}} \\
& =\frac{4 a^{6}-4 a^{5} b-3 a^{4} b^{2}+10 a^{3} b^{3}-3 a^{2} b^{4}-4 a b^{5}+4 b^{6}}{16 s^{2}}
\end{aligned}
$$


where

$$
\begin{aligned}
Q:= & 16 s^{2}\left(a^{2}-a b+b^{2}\right)+4 s\left(3 a^{3}-a^{2} b-a b^{2}+3 b^{3}\right) \\
& +\left(a^{4}+3 a^{3} b-5 a^{2} b^{2}+3 a b^{3}+b^{4}\right), \\
P:= & 4 s\left(a^{2}-a b+b^{2}\right)+\left(2 a^{3}-a^{2} b-a b^{2}+2 b^{3}\right) .
\end{aligned}
$$

By elementary calculations we obtain $|P|<12 s|a|^{2}+6|a|^{3}<12.001 s|a|^{2}$. Using Lemma 5.1 we obtain

$$
\left|(P X-Q Y)-Y\left(P \bar{\alpha}_{1}-Q\right)-Y P\left(\alpha_{1}-\bar{\alpha}_{1}\right)\right|<\frac{8|P|}{63.99|Y|^{3} s^{3}} .
$$

Some elementary calculations together with (5.4) yield

$$
\begin{aligned}
|P X-Q Y| & <|Y|\left|P \bar{\alpha}_{1}-Q\right|+|Y|\left|P\left(\alpha_{1}-\bar{\alpha}_{1}\right)\right|+|Y||P| \frac{10.24|a|^{8}}{s^{7}} \\
& <|Y|\left(2 \frac{|a|^{6}}{s^{2}}+\frac{2.82|a|^{6}}{s^{2}}+\frac{122.9|a|^{10}}{s^{6}}\right)<|Y| \frac{4.83|a|^{6}}{s^{2}} .
\end{aligned}
$$

Since $P X-Q Y \in \frac{1}{256} \mathbb{Z}$, we have $P X-Q Y=0$ provided $|Y| \leq \frac{s^{2}}{1236.48|a|^{6}}$. Inserting this relation in $F(X, Y)= \pm 1$ yields

$$
Y^{4}\left(256 s^{4} A+R_{1}\right)= \pm P^{4}
$$

where

$$
A=\left(a^{2}-a b+b^{2}\right)^{3}\left(a^{6}-3 a^{5} b+9 a^{4} b^{2}-13 a^{3} b^{3}+9 a^{2} b^{4}-3 a b^{5}+b^{6}\right) .
$$

For $a_{0}, a_{1}>0$ we have

$$
\begin{aligned}
X^{2}-a_{0} X Y+a_{1} Y^{2} & =\left(\frac{a_{0}}{2 \sqrt{a_{1}}} X-\sqrt{a_{1}} Y\right)^{2}+X^{2}\left(1-\frac{a_{0}^{2}}{4 a_{1}}\right) \\
& \geq X^{2}\left(1-\frac{a_{0}^{2}}{4 a_{1}}\right) .
\end{aligned}
$$

Therefore we find the estimation

$$
\begin{aligned}
A & =\left(a^{2}-a b+b^{2}\right)^{3}\left(a^{6}-3 a^{5} b+9 a^{4} b^{2}-13 a^{3} b^{3}+9 a^{2} b^{4}-3 a b^{5}+b^{6}\right) \\
& >\frac{27}{64}|a|^{6}\left(a^{2}-a b+5.48 b^{2}\right)\left(a^{2}-0.183 a b+0.182 b^{2}\right)\left(a^{2}-1.818 a b+b^{2}\right) \\
& >|a|^{12} 0.06672 .
\end{aligned}
$$

Furthermore, $R_{1}$ is some expression of lower terms that one can estimate by $\left|R_{1}\right|<199552 s^{3}|a|^{13}+119216 s^{2}|a|^{14}+36904 s|a|^{15}+6597|a|^{16}<2 \cdot 10^{5} s^{3}|a|^{13}$.

Therefore we receive from (5.7) and (5.4)

$$
|Y|^{4}\left|256 s^{4} A+R_{1}\right|>|Y|^{4} 17.09 s^{4}|a|^{12}>0.208 s^{8}|a|^{4} .
$$

On the other hand

$$
|P|^{4}<20743 s^{4}|a|^{8}
$$


which yields a contradiction to (5.7) if $s>18|a|$. Therefore we have proved $|Y|>\frac{s^{2}}{1236.48|a|^{6}}$, i.e. the first case. The proof of the other cases is similar and we will discuss them less detailed.

CASE 2: For this case we use the approximation

$$
\begin{aligned}
\alpha_{2}= & \overbrace{-a+\frac{a^{2}-a b}{4 s}+\frac{-2 a^{3}+a^{2} b+a b^{2}}{16 s^{2}}+\frac{5 a^{4}-2 a^{3} b-a^{2} b^{2}+2 a b^{3}}{64 s^{3}}}^{:=\bar{\alpha}_{2}} \\
& +L\left(\frac{0.1641|a|^{5}}{s^{4}}\right) .
\end{aligned}
$$

For $\bar{\alpha}_{2}$ we obtain a Padé approximation $\frac{Q}{P} \approx \bar{\alpha}_{2}$ with

$$
\begin{aligned}
Q:= & 16 s^{2} a\left(a^{2}-a b+b^{2}\right)-4 s a\left(11 a^{3}+9 a^{2} b+9 a b^{2}+b^{3}\right) \\
& -a\left(13 a^{4}+32 a^{3} b+31 a^{2} b^{2}+17 a b^{3}+7 b^{4}\right), \\
P:= & -16 s^{2}\left(a^{2}-a b+b^{2}\right)+4 s a\left(10 a^{3}+11 a^{2} b+7 a b^{2}+2 b^{3}\right) \\
& +\left(25 a^{4}+30 a^{3} b+29 a^{2} b^{2}+12 a b^{3}+4 b^{4}\right) .
\end{aligned}
$$

Similarly as in the first case we compute

$$
\begin{aligned}
|P X-Q Y| & <|Y|\left|P \bar{\alpha}_{2}-Q\right|+|Y|\left|P\left(\alpha_{2}-\bar{\alpha}_{2}\right)\right|+|Y||P| \frac{303.64|a|^{8}}{s^{5}} \\
& <|Y|\left(\frac{285|a|^{8}}{32 s^{3}}+\frac{7.88|a|^{7}}{s^{2}}+\frac{14576|a|^{10}}{s^{3}}\right)<|Y| \frac{7.9|a|^{7}}{s^{2}} .
\end{aligned}
$$

Therefore $P X-Q Y=0$ provided $|Y| \leq \frac{s^{2}}{8089.6|a|^{7}}$. From $F(X, Y)= \pm 1$ we obtain now

$$
Y^{4}\left(s^{5} A+R_{1}\right)= \pm P^{4},
$$

with

$$
\begin{aligned}
|A|= & 1024 a^{2}(a-b)^{2}\left(a^{2}-a b+b^{2}\right)^{4}\left|14 a^{3}+9 a^{2} b+7 a b^{2}+5 b^{3}\right| \geq \frac{81}{256}|a|^{10}, \\
\left|R_{1}\right| \leq & 5500416 s^{4}|a|^{16}+3231360 s^{3}|a|^{17}+1588768 s^{2}|a|^{18} \\
& +4184008 s|a|^{19}+1966342|a|^{20}<s^{4}|a|^{16} 5.51 \cdot 10^{6}, \\
|P|^{4} \leq & 5.31 \cdot 10^{6} s^{8}|a|^{8} .
\end{aligned}
$$

Comparing the bounds from the right hand side and left hand side of (5.10), we find

$$
0.002 s^{9}|a|^{2}<5.31 \cdot 10^{6} s^{8}|a|^{8},
$$

which is a contradiction for $s>2.66 \cdot 10^{9}|a|^{6}$. 
CASE 3: Now we use the following approximation

$$
\begin{aligned}
\alpha_{3}= & \overbrace{-b+\frac{-a b+b^{2}}{4 s}+\frac{a^{2} b+a b^{2}-2 b^{3}}{16 s^{2}}+\frac{-2 a^{3} b-a^{2} b^{2}-2 b^{3} a+5 b^{4}}{64 s^{3}}}^{:=\bar{\alpha}_{3}} \\
& +L\left(\frac{0.1641|a|^{5}}{s^{4}}\right) .
\end{aligned}
$$

Applying Padé's theorem to $\bar{\alpha}_{3}$, we obtain an approximation $\frac{Q}{P} \approx \bar{\alpha}_{3}$ with

$$
\begin{aligned}
Q:= & 16 s^{2} b\left(a^{2}-a b+b^{2}\right)-4 s b\left(a^{3}+9 a^{2} b+9 a b^{2}+11 b^{3}\right) \\
& -b\left(7 a^{4}+17 a^{3} b+31 a^{2} b^{2}+32 a b^{3}+13 b^{4}\right), \\
P:= & -16 s^{2}\left(a^{2}-a b+b^{2}\right)+4 s a\left(2 a^{3}+7 a^{2} b+11 a b^{2}+10 b^{3}\right) \\
& +\left(4 a^{4}+12 a^{3} b+29 a^{2} b^{2}+30 a b^{3}+25 b^{4}\right) .
\end{aligned}
$$

Similarly as in the first case, we obtain

$$
\begin{aligned}
|P X-Q Y| & <|Y|\left|P \bar{\alpha}_{3}-Q\right|+|Y|\left|P\left(\alpha_{3}-\bar{\alpha}_{3}\right)\right|+|Y||P| \frac{488.57|a|^{8}}{s^{5}} \\
& <|Y|\left(\frac{285|a|^{8}}{32 s^{3}}+\frac{7.88|a|^{7}}{s^{2}}+\frac{23452|a|^{10}}{s^{3}}\right)<|Y| \frac{7.9|a|^{7}}{s^{2}} .
\end{aligned}
$$

Therefore $P X-Q Y=0$ provided $|Y| \leq \frac{s^{2}}{8089.6|a|^{7}}$. If we insert this relation in $F(X, Y)= \pm 1$, we get

$$
Y^{4}\left(s^{5} A+R_{1}\right)= \pm P^{4}
$$

with

$$
\begin{aligned}
|A|= & 1024 b^{2}(a-b)^{2}\left(a^{2}-a b+b^{2}\right)^{4}\left|5 a^{3}+7 a^{2} b+9 a b^{2}+14 b^{3}\right| \geq \frac{18225}{65536}|a|^{10}, \\
\left|R_{1}\right| \leq & 5500416 s^{4}|a|^{16}+3231360 s^{3}|a|^{17}+1588768 s^{2}|a|^{18} \\
& +4184008 s|a|^{19}+1966342|a|^{20}<s^{4}|a|^{16} 5.51 \cdot 10^{6} \\
|P|^{4} \leq & 5.31 \cdot 10^{6} s^{8}|a|^{8} .
\end{aligned}
$$

This time we deduce from (5.12)

$$
0.00153 s^{9}|a|^{2}<5.31 \cdot 10^{6} s^{8}|a|^{8},
$$

a contradiction, provided $s>3.5 \cdot 10^{9}|a|^{6}$.

CASE 4: In the last case we use the method of Padé approximation twice. First we use the following approximation

$$
\alpha_{4}=\overbrace{\frac{a b}{4 s}-\frac{a^{2} b+a b^{2}}{16 s^{2}}}^{:=\bar{\alpha}_{4}}+L\left(\frac{0.235|a|^{4}}{s^{3}}\right)
$$


and by Padé's theorem we obtain an approximation $\frac{Q}{P} \approx \bar{\alpha}_{4}$ with

$$
\begin{aligned}
& Q:=a b, \\
& P:=4 s+a+b .
\end{aligned}
$$

Similarly as in the first case we obtain

$$
\begin{aligned}
|P X-Q Y| & <|Y|\left|P \bar{\alpha}_{4}-Q\right|+|Y|\left|P\left(\alpha_{4}-\bar{\alpha}_{4}\right)\right|+|Y||P| \frac{303.64|a|^{8}}{s^{5}} \\
& <|Y|\left(\frac{|a|^{4}}{4 s^{2}}+\frac{0.95|a|^{4}}{s^{2}}+\frac{1214.9|a|^{8}}{s^{4}}\right)<|Y| \frac{1.21|a|^{4}}{s^{2}}
\end{aligned}
$$

Therefore $P X-Q Y=0$ provided $|Y| \leq \frac{s^{2}}{19.36|a|^{4}}$. This relation together with $F(X, Y)= \pm 1$ yields

$$
Y^{4}\left(s^{2} A+R_{1}\right)= \pm P^{4}
$$

where

$$
\begin{aligned}
|A| & =16 a^{2} b^{2}\left(a^{2}-a b+b^{2}\right) \geq 12|a|^{2}, \\
\left|R_{1}\right| & \leq 24 s|a|^{7}+9|a|^{8}>24.001 s|a|^{7}, \\
|P|^{4} & \leq 257 s^{4} .
\end{aligned}
$$

From (5.14) we deduce

$$
0.08 s^{6}<257 s^{4}|a|^{6}
$$

a contradiction provided $s>57|a|^{3}$. Therefore we may assume $|Y|>\frac{s^{2}}{19.36|a|^{4}}$.

For the second application of Padé approximations we use

$$
\begin{aligned}
\alpha_{4}= & \overbrace{\frac{a b}{4 s}-\frac{a^{2} b+a b^{2}}{16 s^{2}}+\frac{2 a^{3} b+a^{2} b^{2}+2 a b^{3}}{64 s^{3}}-\frac{5 a^{4} b+2 a^{3} b^{2}+2 a^{2} b^{3}+5 a b^{4}}{256 s^{4}}}^{:=\bar{\alpha}_{4}} \\
& +L\left(\frac{0.1231|a|^{6}}{s^{5}}\right) .
\end{aligned}
$$

Therefore we find an approximation $\frac{Q}{P} \approx \bar{\alpha}_{4}$ with

$$
\begin{aligned}
Q:= & 4 a b s\left(a^{2}-a b+b^{2}\right)+2 a b\left(2 a^{3}-a^{2} b-a b^{2}+2 b^{3}\right), \\
P:= & 16 s^{2}\left(a^{2}-a b+b^{2}\right)+4 s\left(3 a^{3}-a^{2} b-a b^{2}+3 b^{3}\right) \\
& +\left(a^{4}+3 a^{3} b-5 a^{2} b^{2}+3 a b^{3}+b^{4}\right) .
\end{aligned}
$$

Similarly as in the first case we obtain

$$
\begin{aligned}
&|P X-Q Y|<|Y|\left|P \bar{\alpha}_{4}-Q\right|+|Y|\left|P\left(\alpha_{4}-\bar{\alpha}_{4}\right)\right|+|Y||P| \frac{281670|a|^{16}}{s^{9}} \\
&<|Y|\left(1.02 \frac{|a|^{8}}{s^{3}}+\frac{5.91|a|^{8}}{s^{3}}+\frac{1.36 \cdot 10^{7}|a|^{18}}{s^{7}}\right)<|Y| \frac{6.94|a|^{8}}{s^{3}} .
\end{aligned}
$$


From (5.15) we deduce $P X-Q Y=0$, if $|Y| \leq \frac{s^{3}}{7107|a|^{8}}$. Let us insert this relation into the original Thue equation $F(X, Y)= \pm 1$. Then

$$
Y^{4}\left(s^{4} A+R_{1}\right)= \pm P^{4},
$$

with

$$
\begin{aligned}
|A|= & 256 a^{2} b^{2}\left(a^{2}-a b+b^{2}\right)^{3} \\
& \times\left(a^{6}-3 a^{5} b+9 a^{4} b^{2}-13 a^{3} b^{3}+9 a^{2} b^{4}-3 a b^{5}+b^{6}\right)>17.1|a|^{12}, \\
\left|R_{1}\right| \leq & 199552 s^{3}|a|^{17}+119216 s^{2}|a|^{18}+36904 s|a|^{19}+6597|a|^{20} \\
< & s^{3}|a|^{17} 2 \cdot 10^{5} \\
|P|^{4} \leq & 5.31 \cdot 10^{6} s^{8}|a|^{8} .
\end{aligned}
$$

This, combined with (5.16), implies

$$
1.21 \cdot 10^{-4} s^{12}|a|^{-4}<5.31 \cdot 10^{6} s^{8}|a|^{8},
$$

a contradiction, since we assume $s>3.5 \cdot 10^{9}|a|^{9}>458|a|^{3}$.

If $a$ and $b$ are given, one may obtain better results than those proved in Proposition 5.2. Indeed, one only has to apply the method of Padé approximations successively to obtain results of the form $|Y|>s^{\mu} c(a, b, \mu)$ for some $\mu>2$. In the general case the difficulty to find optimal or even useful estimations rapidly grows. Observe that the "technical" bound $c_{0}=3.5 \cdot 10^{9}$ in Proposition 5.2 is quite large. In the case of $a, b \in \mathbb{Z}$ the "technical" bound actually exceeds the bound that one obtains by comparing lower and upper bounds for $\log |Y|$ (cf. (5.17) and Theorem 7.1).

Corollary 5.3. Let $(X, Y)$ be a solution to (1.4). Then $|Y| \leq 1$ provided

$$
s>7.23 \cdot 10^{10}|a|^{\frac{29+\sqrt{241}}{4}} .
$$

Proof. Suppose $(X, Y)$ is a solution to (1.4) with $|Y|>1$. Let us compare the bounds from Propositions 4.3 and 5.2 and assume $s>c_{1}|a|^{9+r}$ with $c_{1}>\max \left(3.5 \cdot 10^{9}, c_{0}(r)\right)$ (cf. Proposition 4.3). Then we obtain

$$
\begin{gathered}
\left(2-\frac{7}{9+r}\right) \log s+\frac{7}{9+r} \log c_{1}-\log 8089.6<\log |Y| \\
<\frac{24+4 r}{(9+r) r}\left(\log s-\log c_{1}\right)+\frac{10.71}{2} \frac{12+2 r}{r}+0.144
\end{gathered}
$$

and therefore

(5.17)

$$
\frac{2 r^{2}+7 r-24}{r(r+9)} \log s+\frac{11 r+24}{r+9} \log c_{1}<\frac{10.71}{2} \frac{12+2 r}{r}+0.144+\log 8089.6 .
$$

The coefficient of $\log s$ is positive if $r>\frac{-7+\sqrt{241}}{4}$ and (5.17) fails provided $s$ is large enough. Suppose now $r=\frac{-7+\sqrt{241}}{4}$. Then the coefficient of $\log s$ 
is zero and (5.17) also fails if $c_{1}$ is large enough, i.e. $c_{1}>7.23 \cdot 10^{10}>$ $\max \left(3.5 \cdot 10^{9}, c_{0}(r)\right)$.

\section{The CASE of $|Y|=1$}

In view of Corollary 5.3 it remains to consider the case of $|Y| \leq 1$ in order to prove Theorem 1.1. The case $Y=0$ yields the trivial solutions $(X, Y)=( \pm 1,0)$. Therefore we are left to the four cases $Y= \pm 1$ and $\mu= \pm 1$ with mixed signs. Since with $(X, Y)$ also $(-X,-Y)$ is a solution to (1.4) we only have to check the cases $Y=1$ and $\mu= \pm 1$.

Let us consider first the case $Y=1$ and $\mu=1$. Thue equation (1.4) reduces to

$$
P(X)=X^{4}-4 s X^{3}-(2 a b+4 s(a+b)) X^{2}-4 a b s X+a^{2} b^{2}-1=0 .
$$

Since $F(X, 1)=P(X)+1$, we deduce that the roots of $P(X)$ and $F(X, 1)$ are close together. Therefore we want to prove that the roots of $P(X)$ lie in the disjoint intervals

$$
\begin{aligned}
& I_{1}:=(4 s+a+b-1 / 8,4 s+a+b+1 / 8), \\
& I_{2}:=(-a-1 / 8,-a+1 / 8), \\
& I_{3}:=(-b-1 / 8,-b+1 / 8) \text { and } \\
& I_{4}:=(-1 / 8,1 / 8) .
\end{aligned}
$$

Let us consider the quantities

$$
\begin{gathered}
-P\left(4 s+a+b-\frac{1}{8}\right) P\left(4 s+a+b+\frac{1}{8}\right)=64 s^{6}+128(a+b) s^{5}+\cdots \\
-P\left(-a-\frac{1}{8}\right) P\left(-a+\frac{1}{8}\right)=\frac{\left(-1+64 a^{2}\right)\left(-1+64(a-b)^{2}\right)}{16384} s^{2}+\cdots>\frac{63 \cdot 3}{16384} a^{2} s^{2}+\cdots \\
-P\left(-b-\frac{1}{8}\right) P\left(-b+\frac{1}{8}\right)=\frac{\left(-1+64 b^{2}\right)\left(-1+64(a-b)^{2}\right)}{16384} s^{2}+\cdots>\frac{168}{16384} a^{2} s^{2}+\cdots \\
-P\left(-\frac{1}{8}\right) P\left(\frac{1}{8}\right)=\frac{\left(-1+64 a^{2}\right)\left(-1+64 b^{2}\right)}{16384} s^{2}+\cdots>\frac{63 \cdot 3}{16384} a^{2} s^{2}+\cdots
\end{gathered}
$$

which yield that if $s$ is large enough then each root of $P$ lies in one of the intervals $I_{1}, I_{2}, I_{3}$ or $I_{4}$. A more detailed analysis yields that $s>130.4|a|^{3}$ is adequate (also for the case $\mu=-1$ ). Therefore the only integral solutions may be $X=4 s+a+b,-a,-b$ or $X=0$. Inserting in (1.4) yields

$$
\begin{gathered}
16\left(a^{2}-a b+b^{2}\right) s^{2}+4\left(2 a^{3}+a^{2} b+a b^{2}+2 b^{3}\right) s \\
+\left(a^{4}+2 a^{3} b+3 a^{2} b^{2}+2 a b^{3}+b^{4}\right)=1, \\
a^{2}(a-b)^{2}=1, \\
b^{2}(a-b)^{2}=1, \\
a^{2} b^{2}=1,
\end{gathered}
$$

respectively. The first equation fails if $s$ is too "large", i.e. $s>2.36|a|$. The other equations only yield solutions listed in Table 1.

Similar arguments apply for $\mu=-1$. Observe that in this case there are no solutions. Therefore we have proved the following proposition: 
Proposition 6.1. Let $(X, Y)$ be a solution to (1.4) with $|Y|=1$ and let $s>130.4|a|^{3}$. Then the solution $(X, Y)$ is listed in Theorem 1.1 or Table 1.

Combining Corollary 5.3 and Proposition 6.1 we immediately obtain Theorem 1.1 .

\section{Some EXAmples}

First let us state a theorem that one may obtain by recomputing the proof of Theorem 1.1.

TheOREm 7.1. If $a, b \in \frac{1}{2} \mathbb{Z}$, respectively $a, b \in \mathbb{Z}$. Then Theorem 1.1 also holds for $s>6.33 \cdot 10^{7}|a|^{\frac{29+\sqrt{241}}{4}}$, respectively $s>1.07 \cdot 10^{5}|a|^{\frac{29+\sqrt{241}}{4}}$.

Let us now consider four examples to illustrate Theorems 1.1 and 7.1:

- Let $a=-2$ and $b=1 / 2$. Then we have the Thue equation

$$
X^{4}-4 s X^{3} Y+(6 s+2) X^{2} Y^{2}+4 s X Y^{3}+Y^{4}= \pm 1 .
$$

Let $(X, Y)$ be a solution to $(7.1)$. Then $(X, Y)=(0, \pm 1)$ or $(X, Y)=$ $( \pm 1,0)$ if $s>1.42 \cdot 10^{11}$. Note that Thue equation (7.1) has been solved for all $s \geq 0$ in the case of the +-sign by Dujella and Jadrijević [9].

- Let $a=1$ and $b=-1$. Then $( \pm 1,0)$ and $(0, \pm 1)$ are the only solutions to the Thue equation

$$
X^{4}-4 s X^{3} Y+2 X^{2} Y^{2}+4 s X Y^{3}+Y^{4}= \pm 1
$$

provided $s>1.07 \cdot 10^{5}$.

- Let $a=5 / 2, b=2$ and $s>1.71 \cdot 10^{12}$. Then the Diophantine equation

$$
X^{4}-4 s X^{3} Y-(10+18 s) X^{2} Y^{2}-20 s X Y^{3}+25 Y^{4}= \pm 1
$$

has only the solutions $( \pm 1,0),(2,-1)$ and $(-2,1)$.

- Let $a=4$ and $b=-\frac{13}{4}$. Then $(X, Y)=( \pm 1,0)$ is the only solution to Thue equation

$$
X^{4}-4 s X^{3} Y+(26-3 s) X^{2} Y^{2}+52 s X Y^{3}+169 Y^{4}= \pm 1
$$

if we assume $s>3.64 \cdot 10^{17}$.

All four examples have been solved using Theorem 1.1, respectively Theorem 7.1 .

In most cases one could obtain sharper bounds for $s$, if one would apply the method of Padé approximations several times more. However Dujella and Jadrijević [9] used the "congruence method" in order to obtain a sharp estimate for $s$ in the case of equation (7.1). In order to apply this powerful method, we start with system (3.6) and multiply the first equation by $b$ and 
put $k=s b+\frac{a b}{2}$ and similarly we multiply the second equation by $a$ and put $k=s a+\frac{a b}{2}$. In both cases we obtain a Pellian equation of the form

$$
X^{2}\left(k+\frac{a b}{2}\right)-Y^{2}\left(k-\frac{a b}{2}\right)=\mu a b .
$$

Note that the coefficients of $(7.5)$ need not to be integers. The congruence method essentially depends on finding a "fundamental solution" to (7.5), i.e. in the cases above we have to find a fundamental solution corresponding to

$$
\begin{aligned}
X^{2}-\left(4 k^{2}-1\right) Y^{2} & =1, \\
X^{2}-\left(4 k^{2}-1\right) Y^{2} & =1, \\
X^{2}-\left(4 k^{2}-25\right) Y^{2} & =1, \\
X^{2}-\left(16 k^{2}-676\right) Y^{2} & =1,
\end{aligned}
$$

respectively. Note that the Pell equations above have integral coefficients. The first two cases yield the fundamental solution $2 k+\sqrt{4 k^{2}-1}$. For the other cases no parameterized fundamental solutions are known and we cannot apply the powerful "congruence method" in those cases. A close look on (7.5) shows that only in the case of $|a b|=1$ and $a, b \in \frac{1}{2} \mathbb{Z}$, or $|a b|=2$ and $a, b \in \mathbb{Z}$, we can find parameterized fundamental solutions. In all other cases no parameterized fundamental solutions are known.

\section{REFERENCES}

[1] A. Baker, Linear forms in the logarithms of algebraic numbers I, II, III, Mathematika 13 (1966), 204-216; ibid. 14 (1967), 102-107; ibid. 14 (1967), 220-228.

[2] A. Baker, Contributions to the theory of Diophantine equations I. On the representation of integers by binary forms, Philos. Trans. Roy. Soc. London Ser. A 263 (1967/1968), 173-191.

[3] A. Baker, Linear forms in the logarithms of algebraic numbers IV, Mathematika 15 (1968), 204-216.

[4] A. Baker and H. Davenport, The equations $3 x^{2}-2=y^{2}$ and $8 x^{2}-7=z^{2}$, Quart. J. Math. Oxford Ser. (2) 20 (1969), 129-137.

[5] M. A. Bennett, On the number of solutions of simultaneous Pell equations, J. Reine Angew. Math. 498 (1998), 173-199.

[6] Yu. Bilu and G. Hanrot, Solving Thue equations of high degree, J. Number Theory 60 (1996), 373-392.

[7] Y. Bugeaud and K. Györy, Bounds for the solutions of Thue-Mahler equations and norm form equations, Acta Arith. 74 (1996), 273-292.

[8] G. V. Chudnovsky, On the method of Thue-Siegel, Ann. of Math. (2) 117 (1983), 325-382.

[9] A. Dujella and B. Jadrijević, A parametric family of quartic Thue equations, Acta Arith. 101 (2002), 159-170.

[10] A. Dujella and B. Jadrijević, A family of quartic Thue inequalities, Acta Arith. 111 (2004), 61-76.

[11] S. Lang, Algebra, Springer-Verlag, New York, 2002.

[12] K. Mahler, Zur Approximation algebraischer Zahlen (I), Math. Ann. 107 (1933), 691-730. 
[13] L. J. Mordell, Diophantine equations, Academic Press, London, 1969.

[14] E. Thomas, Complete solutions to a family of cubic Diophantine equations, J. Number Theory 34 (1990), 235-250.

[15] A. Thue, Über Annäherungswerte algebraischer Zahlen, J. Reine und Angew. Math. 135 (1909), 284-305.

[16] A. Thue, Berechnung aller Lösungen gewisser Gleichungen von der Form $a x^{r}-b y^{r}=$ $f$, Vid. Skrivter I Mat.- Naturv. Klasse 4 (1918), 1-9.

[17] N. Tzanakis, Explicit solution of a class of quartic Thue equations, Acta Arith. 64 (1993), 271-283.

[18] N. Tzanakis and B. M. M. de Weger, On the practical solution of the Thue equation, J. Number Theory 31 (1989), 99-132.

Institut für Mathematik A

Technische Universität Graz

Steyrergasse 30

A-8010 Graz

Austria

E-mail: ziegler@finanz.math.tugraz.at

Received: 15.4 .2005 .

Revised: 23.8.2005. 